\title{
A mathematical model for the effect of anti-angiogenic therapy in the treatment of cancer tumours by chemotherapy
}

\author{
S.T.R. Pinho ${ }^{\mathrm{a}, *}$, F.S. Bacelar ${ }^{\mathrm{a}, \mathrm{b}}$, R.F.S. Andrade ${ }^{\mathrm{a}}$, H.I. Freedman ${ }^{\mathrm{c}}$ \\ a Instituto de Física, Universidade Federal da Bahia, 40210-340, Salvador, Brazil \\ ${ }^{\mathrm{b}}$ IFISC(CSIC-UIB) Instituto de Física Interdisciplinar y Sistemas Complejos, Campus Universitat Illes Balears, E-07122 Palma de Mallorca, Spain \\ ${ }^{\mathrm{c}}$ Department of Mathematical and Statistical Sciences, University of Alberta, Edmonton, Alberta, Canada T6G 2G1
}

\section{A R T I C L E I N F O}

\section{Article history:}

Received 3 August 2011

Accepted 29 July 2012

\section{Keywords:}

Differential equations model

Cancer therapy

Angiogenesis

\begin{abstract}
A B S T R A C T
A model consisting of a system of five ordinary differential equations to simulate the interactions between normal cells, cancer cells, endothelial cells, chemotherapy agent and anti-angiogenic agent in tumour growth is developed. By a partial analysis of the cancerfree subspace, it is shown how the anti-angiogenic agent may help the chemotherapy agent in controlling the cancer. This is illustrated by numerical examples and bifurcation diagrams.
\end{abstract}

(c) 2012 Elsevier Ltd. All rights reserved.

\section{Introduction}

Neoplastic diseases are responsible for $12 \%$ of deaths around the world. They puzzle researchers of several disciplines with ever new and intriguing challenges in relation to the dynamics and control of tumour growth. In recent years, multiscale models for cancer therapies have been of great interest [1,2]. In order to analyse vascular tumour growth for which the number of cells is very large (tumour diameter $D>2 \mathrm{~mm}$ ), it is reasonable to base our model on a continuum assumption, namely that cell growth is an essentially continuous process. Thus cell growth models have mostly consisted of differential equations [3,4], and more specifically ordinary differential equations (ODEs) in the case of modelling tumour growth subjected to therapy [5-7]. In this case the spatial heterogeneity of the cells is neglected as a first approximation, assuming homogeneous distributions of the cells in a single well-mixed tissue, in such a way that the therapy agents act on the cells' compartments.

Frequently the interaction between normal cells (NCs) and cancer cells (CCs) is thought of as a competition for bodily nutrients [8], and the most common chemical treatment (chemotherapy) as a killing relationship between the chemotherapy agent (CA) and both NCs and CCs, as assumed by some of us in [9]. We consider CA to be a cell-cycle non-specific chemotherapeutic drug (e.g. cyclophosphamide [10]) that does not distinguish proliferating and non-proliferating cells.

In the specific case of malignant tumours that reach a certain size, however, CCs create mechanisms to provide themselves with the necessary nutrients for their rapid growth [11,12]. This means that CCs induce substances that lead to the development and growth of an additional endothelial cell (EC) population [13,14], which have several effects on the NCs and CCs. Firstly the ECs cause blood cells to proliferate and to bring oxygen and nutrients to the tumour: this mechanism is known as tumour angiogenesis [15]. Hence the ECs effectively increase the carrying capacity of the CCs [16] due to neo-

\footnotetext{
* Correspondence to: Instituto de Física, Universidade Federal da Bahia, Campus Universitário de Ondina, 40210-340, Salvador, Brazil. Tel.: +55 71 32836658; fax: +55 7132357730 .

E-mail addresses: suani@ufba.br (S.T.R. Pinho), fbacelar@ufba.br (F.S. Bacelar), randrade@ufba.br (R.F.S. Andrade), hfreedma@math.ualberta.ca (H.I. Freedman).
} 
vascularization. Since the tumour induces the occurrence of angiogenesis in its neighbourhood, it is reasonable to neglect the effect of ECs in the carrying capacity of NCs.

In order to affect the angiogenic process, an anti-angiogenic agent (AA) is introduced in many treatments $[17,18]$. It happens that AA is particularly efficient for slow growing solid tumours [19]. This has a saturated mass action effect in reducing the growth rate of ECs. In the case of co-administration of AA and CA, AA is found to increase the efficiency of CA [20] since it destroys the excess of ECs that contributes to the formation of an irregular structure of blood vessels, normalizing the tumour vasculature. However, the vasculature should not be completely destroyed as a certain amount of vasculature is necessary to allow for the flux of CA. Therefore, the concomitant presence of both ECs and AA leads to a net result of making the oxygen and chemotherapy delivery more efficient [21,22].

With the purpose of analysing the response of chemo-resistant tumours to the co-administration of CA and AA, it is reasonable to neglect the CA action on ECs in comparison to that of AA on the same ECs. Even the CA administration in the case of chemo-resistant tumours seems to have a negligible anti-angiogenic side effect [23]. It is important to point out that, although the change from the conventional chemotherapy schedule to almost continuous schedule may increase the anti-angiogenic side effect of CA, this is not so important as the killing effect of AA on ECs and enhanced killing action of CA on CCs.

In order to model the above considerations, we develop below a system of five ODEs for the interactions between the three compartments of cells, NCs, CCs and ECs, and the two compartments of agents, CA and AA. We assume continuous infusion of the agents to simulate the response to chemo-resistant tumours. Of course, a complete analysis of such a model, or even of its 3- and 4-dimensional subspaces is mathematically intractable. Note that other models have been proposed to analyse a combined CA+AA treatment: in [1], a multiscale model is considered, in which CA treatment follows AA treatment; in [24], an ODE model that does not consider an NC compartment is investigated.

As well-known from clinical observations, sometimes chemotherapy is able to eradicate the cancer possibly after surgery and/or other treatments (eg. immunotherapy, radiotherapy), but sometimes not. It is also generally known that AA itself cannot drive cancer to extinction $[1,22,25]$. The purpose of this paper is to show that the AA may aid the CA in cancer treatment and, in fact, may even facilitate a "reversal of outcome". An example of this is given in [26] where certain drug resistant tumours in mice are shown to respond positively to a combined therapy eliminating the tumour. Further, such a combined therapy is recommended for humans, for instance, in the case of colorectal tumours [27]. Hence this paper is able to add credence to certain (possibly controversial) experimental results.

We may anticipate the two main issues and contributions of this work. In the first place, the co-administration of CA and $\mathrm{AA}$ is able to eliminate the tumour in the case of drug-resistant tumours, promoting a reversal of outcome. Our analysis indicates that this positive scenario may be reached due to the fact that the AA kills ECs, but it also causes an enhancement of the CA killing action on CCs. Secondly, from the modelling point of view, our CA and AA continuous infusion approach gives rise to a discussion about the reduction of time intervals between infusions, which was already discussed from the experimental point of view [26].

We consider the subspaces where the CCs are absent and check for the equilibria (or absence thereof) both in the absence and presence of AA. We refer to such equilibria as the "cure states". We then develop criteria for such cure states to not exist or be unstable in the CC direction in the absence of AA but to be asymptotically stable in the CC direction in the presence of AA. We illustrate this with numerical examples. This gives a partial picture of how AA can aid CA in eradicating CCs (to be elaborated in the discussion section).

The paper is organized as follows. In the next section we state our model and prove some elementary properties. In Section 3, we look at the sub-models in the absence of CA or AA, in Section 4, in the presence of both CA and AA. This is followed in Section 5 with numerical examples and bifurcation diagrams. We end with a discussion in the final section.

\section{The model}

We now present our model of interactions between NCs, CCs, ECs, CA and AA. $X_{i}(t)$ is the concentration of NCs, CCs, and ECs, respectively, for $i=1,2,3 ; Y(t)$ is the concentration of the chemotherapy agent and $W(t)$ is the concentration of the anti-angiogenic agent for $t \geq 0$. The model is based on the considerations discussed in the Introduction, which we summarize below.

(1) Both $X_{1}(t)$ and $X_{2}(t)$ exhibit logistic proliferation rates and compete for available resources.

(2) $X_{3}(t)$ is regulated by the net result of chemical factors produced by $X_{2}(t)$ as well as by a much slower logistic proliferation rate.

(3) $Y(t)$ acts as a killer, with different intensities, of on $X_{1}(t)$ and $X_{2}(t)$, according to a Holling type 2 function [28]. $W(t)$ acts only on $X_{3}(t)$.

(4) $Y(t)$ and $W(t)$ increase due to their continuous infusions into the system [9].

(5) Both $Y(t)$ and $W(t)$ decrease due to its action on the cells and also because of the washout process for both therapies.

(6) The killing action of $Y(t)$ depends on the vascularization $\left(X_{3}(t)\right)$ and on its normalization set up by $W(t)$.

(7) The chemical and anti-angiogenic therapies are applied simultaneously, i.e., by co-administration [26]. 
Hence, we take as our model the following system of ODEs:

$$
\begin{aligned}
& \dot{X}_{1}(t)=\alpha_{1} X_{1}(t)\left[1-\frac{X_{1}(t)}{K_{1}}\right]-Q_{1} X_{1}(t) X_{2}(t)-P_{1}\left(X_{3}(t), W(t)\right) \frac{X_{1}(t) Y(t)}{A_{1}+X_{1}(t)}, \\
& \dot{X}_{2}(t)=\alpha_{2} X_{2}(t)\left[1-\frac{X_{2}(t)}{K_{2}+\Gamma X_{3}(t)}\right]-Q_{2} X_{1}(t) X_{2}(t)-P_{2}\left(X_{3}(t), W(t)\right) \frac{X_{2}(t) Y(t)}{A_{2}+X_{2}(t)}, \\
& \dot{X}_{3}(t)=B X_{2}(t)+\alpha_{3} X_{3}(t)\left[1-\frac{X_{3}(t)}{K_{3}}\right]-\frac{P_{3} X_{3}(t) W(t)}{A_{3}+X_{3}(t)}, \\
& \dot{Y}(t)=\delta-\left[\xi+d_{1} \frac{X_{1}(t)}{A_{1}+X_{1}(t)}+d_{2} \frac{X_{2}(t)}{A_{2}+X_{2}(t)}\right] Y(t), \\
& \dot{W}(t)=\phi-\left[\eta+\frac{d_{3} X_{3}(t)}{A_{3}+X_{3}(t)}\right] W(t)
\end{aligned}
$$

with $^{\circ}=d / d t$ and

$$
P_{i}\left(X_{3}(t), W(t)\right)=P_{i 0}+P_{i 1} X_{3}(t)+P_{i 2} W(t), \quad i=1,2 .
$$

Initial conditions are given by $X_{1}(t=0)=X_{10} \geq 0, X_{2}(t=0)=X_{20} \geq 0, X_{3}(t=0)=X_{30} \geq 0, Y(t=0)=Y_{0} \geq 0$, $W(t=0)=W_{0} \geq 0$.

The model parameters assume positive values and may be interpreted as follows.

- $\alpha_{i}$ and $K_{i}, i=1,2,3$, are the proliferation rates and carrying capacities of $X_{i}$;

- $Q_{i}, i=1,2$, is the competition coefficient between $X_{1}$ and $X_{2}$;

- $B$ is the rate of creation of CCs due to ECs;

- $\Gamma$ is the proportion of ECs responsible for the tumour angiogenesis;

- $P_{i 0}, i=1,2$, is the killing rate of chemotherapy on $X_{i}$ in the absence of $X_{3}$ and $W$ respectively;

- $P_{i j}, i, j=1,2$, is the rate of increased killing on $X_{i}$ by CA per concentration of $X_{3}(j=1)$ and $W(j=2)$;

$-P_{3}$ is the killing rate of anti-angiogenic therapy on $X_{3}$;

- $A_{i}, i=1,2,3$, is the Holling type 2 constant for $X_{i}$;

$-\delta$ and $\phi$ are the respective infusion rates of CA and AA;

- $\xi$ and $\eta$ are, respectively, the washout rates from the system of CA and AA;

- $d_{i}, i=1,2,3$, is the rate at which AA and CA combine with $X_{i}$ (note that $P_{i 0}$ and $d_{i}, i=1,2$ as well as $P_{3}$ and $d_{3}$ may be proportionally related).

We now simplify the model by eliminating three of the parameters. If we let $x_{i}(t)=X_{i}(t) / K_{i}, i=1,2,3, y(t)=Y(t)$ and $w(t)=W(t) ; q_{1}=Q_{1} K_{2}, q_{2}=Q_{2} K_{1}, a_{i}=A_{i} / K_{i}, i=1,2,3, \gamma=\Gamma K_{3} / K_{2}, \beta=B K_{2} / K_{3}, p_{i 0}=P_{i 0} / K_{i}, i=1,2, p_{i 1}=$ $P_{i} K_{3} / K_{i}, i=1,2, p_{i 2}=P_{i 2} / K_{i}, i=1,2, p_{3}=P_{3} / K_{3}$, then our model becomes:

$$
\begin{aligned}
& \dot{x}_{1}(t)=\alpha_{1} x_{1}(t)\left(1-x_{1}(t)\right)-q_{1} x_{1}(t) x_{2}(t)-p_{1}\left(x_{3}(t), w(t)\right) \frac{x_{1}(t) y(t)}{a_{1}+x_{1}(t)}, \\
& \dot{x}_{2}(t)=\alpha_{2} x_{2}(t)\left[1-\frac{x_{2}(t)}{1+\gamma x_{3}(t)}\right]-q_{2} x_{1}(t) x_{2}(t)-p_{2}\left(x_{3}(t), w(t)\right) \frac{x_{2}(t) y(t)}{a_{2}+x_{2}(t)}, \\
& \dot{x_{3}}(t)=\beta x_{2}(t)+\alpha_{3} x_{3}(t)\left[1-x_{3}(t)\right]-\frac{p_{3} x_{3}(t) w(t)}{a_{3}+x_{3}(t)}, \\
& \dot{y}(t)=\delta-\left[\xi+d_{1} \frac{x_{1}(t)}{a_{1}+x_{1}(t)}+d_{2} \frac{x_{2}(t)}{a_{2}+x_{2}(t)}\right] y(t), \\
& \dot{w}(t)=\phi-\left[\eta+\frac{d_{3} x_{3}(t)}{a_{3}+x_{3}(t)}\right] w(t) .
\end{aligned}
$$

with $p_{i}\left(x_{3}(t), w(t)\right)=p_{i 0}+p_{i 1} x_{3}(t)+p_{i 2} w(t) i=1,2$ and $x_{i}(0) \geq 0, i=1,2,3, y(0) \geq 0, w(0) \geq 0$.

It is important to note that, when $x_{2}=0$, the subspace $\left\{\left(x_{1}, 0, x_{3}, y, w\right): x_{1} \geq 0, x_{3} \geq 0, y \geq 0, w \geq 0\right\}$ is invariant under (3). The same is valid for the subspace where $x_{1}=0$ but $x_{2}>0$. Note also that the system (3), which is an autonomous system with differentiable functions, satisfies existence and uniqueness of initial value problems [29,9]. Finally, it is easy to see that, by the invariance of the $x_{1}$ and $x_{2}$ axes, and by the fact that $\dot{x}_{3}(t)_{x_{3}=0} \geq 0, \dot{y}(t)_{y=0}>0, \dot{w}(t)_{w=0}>0$, all solutions with nonnegative initial conditions remain in the nonnegative orthant.

We now show that our system is dissipative, i.e., there exists a compact region in the nonnegative orthant $\mathcal{R}$ such that all solutions initiating in $\mathcal{R}$ remain there, and all solutions initiating outside $\mathcal{R}$ in the nonnegative orthant enter it in finite time. 
Theorem 1. System (3) is dissipative.

Proof. Since the initial conditions are nonnegative, so are the solutions. From the first equation of system (3), it follows that $\dot{x}_{1}(t) \leq \alpha_{1} x_{1}(t)\left(1-x_{1}(t)\right)$.

From the standard Kamke comparison theory we get

$$
\lim _{t \rightarrow \infty} \sup x_{1}(t) \leq 1 \text {. }
$$

Since $d_{i}>0$, with $i=1,2$, we obtain from the fourth equation of system (3):

$$
\dot{y}(t) \leq \delta-\xi y,
$$

which implies

$$
\lim _{t \rightarrow \infty} \sup y(t) \leq \frac{\delta}{\xi} .
$$

Similarly, from the fifth equation of system (3),

$$
\lim _{t \rightarrow \infty} \sup w(t) \leq \frac{\phi}{\eta} \text {. }
$$

After some calculations, the third equation of system (3) leads to:

$$
\lim _{t \rightarrow \infty} \sup x_{3}(t) \leq M_{1},
$$

where

$$
M_{1}=\frac{1}{2}\left(1+\frac{\gamma \beta}{\alpha_{3}}\right)+\frac{1}{2} \sqrt{\left(1+\frac{\beta \gamma}{\alpha_{3}}\right)^{2}+\frac{4 \beta}{\alpha_{3}}} .
$$

From the second equation of system (3),

$$
\lim _{t \rightarrow \infty} \sup x_{2}(t) \leq 1+\gamma M_{1} \text {. }
$$

Hence, the region

$$
\mathcal{R}=\left\{\left(x_{1}, x_{2}, x_{3}, y, w\right) \in \mathbf{R}_{+}^{5} / 0 \leq x_{1} \leq 1,0 \leq x_{2} \leq 1+\gamma M_{1}, 0 \leq x_{3} \leq M_{1}, 0 \leq y \leq \xi^{-1} \delta, 0 \leq w \leq \eta^{-1} \phi\right\}
$$

is an attracting region, proving the property.

As a final comment in this section, we wish to define more precisely what we mean by the 'cancer hypothesis' [9,30]. We actually mean that, in the absence of treatment, CCs always win the competition with NCs for the considered cancer tissue. So, when we state that the 'cancer hypothesis' is valid, we do not take into account the individuals for which the natural immune response is able to win the fight against the disease, even in the absence of any treatment. Biologically, the most common situation leads to the death of the individual. Mathematically, for our model, 'cancer hypothesis' is the condition under which the solution to the system (3), with $Y \equiv 0$ and $W \equiv 0$, satisfies $\lim _{t \rightarrow \infty}\left(x_{1}(t), x_{2}(t)\right)=(0,1)$. Let us note that, in the case of no endothelial cells, the first two equations of system (3) satisfy

$$
\begin{aligned}
& \dot{x}_{1}(t)=\alpha_{1} x_{1}(t)\left(1-x_{1}(t)\right)-q_{1} x_{1}(t) x_{2}(t), \\
& \dot{x}_{2}(t)=\alpha_{2} x_{2}(t)\left(1-x_{2}(t)\right)-q_{2} x_{1}(t) x_{2}(t)
\end{aligned}
$$

in which we must have, if $x_{1}(0) \geq 0, x_{2}(0)>0$, then $\lim _{t \rightarrow \infty}\left(x_{1}(t), x_{2}(t)\right)=(0,1)$ holds. From [31], this implies that the 'cancer hypothesis' consists in the conditions

$$
\alpha_{1}-q_{1}<0 \text { and } \alpha_{2}-q_{2}>0,
$$

which we assume to be valid throughout this paper. The conditions (5) guarantee the global stability of the equilibrium with no NCs in the absence of treatment. Since the parameter $q_{2}$ is related to the immunological response of NCs in the presence of CCs [8], it is consistent that its values are limited by conditions (5). Moreover, note that they are sufficient conditions in the presence of ECs.

In the next sections, we perform a comparative study of the action of therapies based on analytical and numerical results for the model.

\section{Analytical results in sub-models}

In this section we consider two sub-models, each of them involving only one cancer-destroying agent. 


\subsection{No chemotherapy}

In this case $y \equiv 0$ (and $\delta=0$ ). Now the model becomes

$$
\begin{aligned}
& \dot{x}_{1}(t)=\alpha_{1} x_{1}(t)\left(1-x_{1}(t)\right)-q_{1} x_{1}(t) x_{2}(t) \\
& \dot{x}_{2}(t)=\alpha_{2} x_{2}(t)\left[1-\frac{x_{2}(t)}{1+\gamma x_{3}(t)}\right]-q_{2} x_{1}(t) x_{2}(t) \\
& \dot{x_{3}}(t)=\beta x_{2}(t)+\alpha_{3} x_{3}(t)\left[1-x_{3}(t)\right]-\frac{p_{3} x_{3}(t) w(t)}{a_{3}+x_{3}(t)} \\
& \dot{w}(t)=\phi-\left[\eta+\frac{d_{3} x_{3}(t)}{a_{3}+x_{3}(t)}\right] w(t),
\end{aligned}
$$

with

$$
x_{i}(0) \geq 0, \quad i=1,2,3, \quad w(0) \geq 0 .
$$

From the first two of these equations we get

$$
\begin{aligned}
& \dot{x}_{1}(t)=\alpha_{1} x_{1}(t)\left(1-x_{1}(t)\right)-q_{1} x_{1}(t) x_{2}(t), \\
& \dot{x}_{2}(t) \geq \alpha_{2} x_{2}(t)\left(1-x_{2}(t)\right)-q_{2} x_{1}(t) x_{2}(t) .
\end{aligned}
$$

Since by (5) solutions of (4) satisfy $\lim _{t \rightarrow \infty} x_{1}(t)=0$, then by Theorem 1.7.1 of [32], $\lim _{t \rightarrow \infty} x_{1}(t)=0$ for system (8). Further $\lim \inf _{t \rightarrow \infty} x_{2}(t)>0$ by the same theorem in [32].

Remark 1. AA by itself is not able to eliminate the cancer cells.

\subsection{No anti-angiogenic therapy}

In this case $w \equiv 0$ (and $\phi=0$ ). Now the model becomes

$$
\begin{aligned}
& \dot{x}_{1}(t)=\alpha_{1} x_{1}(t)\left(1-x_{1}(t)\right)-q_{1} x_{1}(t) x_{2}(t)-p_{1}\left(x_{3}(t)\right) \frac{x_{1}(t) y(t)}{a_{1}+x_{1}(t)} \\
& \dot{x}_{2}(t)=\alpha_{2} x_{2}(t)\left[1-\frac{x_{2}(t)}{1+\gamma x_{3}(t)}\right]-q_{2} x_{1}(t) x_{2}(t)-p_{2}\left(x_{3}(t)\right) \frac{x_{2}(t) y(t)}{a_{2}+x_{2}(t)} \\
& \dot{x_{3}}(t)=\beta x_{2}(t)+\alpha_{3} x_{3}(t)\left[1-x_{3}(t)\right] \\
& \dot{y}(t)=\delta-\left[\xi+d_{1} \frac{x_{1}(t)}{a_{1}+x_{1}(t)}+d_{2} \frac{x_{2}(t)}{a_{2}+x_{2}(t)}\right] y(t),
\end{aligned}
$$

with

$$
x_{i}(0) \geq 0, \quad i=1,2,3, \quad y(0) \geq 0 .
$$

Here we are interested to know whether a cure state exists or not. Hence we look for the equilibrium $\hat{F}\left(\hat{x}_{1}, 0, \hat{x}_{3}, \hat{y}\right)$ with $\hat{x}_{1}>0$. This requires us to solve the algebraic system

$$
\left\{\begin{array}{l}
\alpha_{1}\left(1-x_{1}\right)-\left(p_{10}+p_{11} x_{3}\right) y /\left(a_{1}+x_{1}\right)=0 \\
\alpha_{3} x_{3}\left(1-x_{3}\right)=0 \\
\delta-\xi y-d_{1} x_{1} /\left(a_{1}+x_{1}\right) y=0
\end{array}\right.
$$

Then clearly $\hat{x}_{3}=0$ or $\hat{x}_{3}=1$. However, in the $x_{1}-x_{3}-y$ subspace, $x_{3}(t) \equiv 0$ only if $x_{3}(0)=0$, otherwise $\lim _{t \rightarrow \infty}$ $x_{3}(t)=1$. The case of no endothelial cells and no anti-angiogenic agent has been analysed previously [9]. In any case, if there are no endothelial cells, there will be no angiogenic process and hence no need for $w$. Hence we consider only the case $x_{3}=1$.

Now our problem reduces to solving the system of two equations:

$$
\begin{aligned}
& \hat{y}=\frac{\alpha_{1}\left(1-\hat{x}_{1}\right)\left(a_{1}+\hat{x}_{1}\right)}{p_{10}+p_{11}} \\
& \hat{y}=\frac{\left(a_{1}+\hat{x}_{1}\right) \delta}{\left(\xi+d_{1}\right) \hat{x}_{1}+\xi a_{1}} .
\end{aligned}
$$



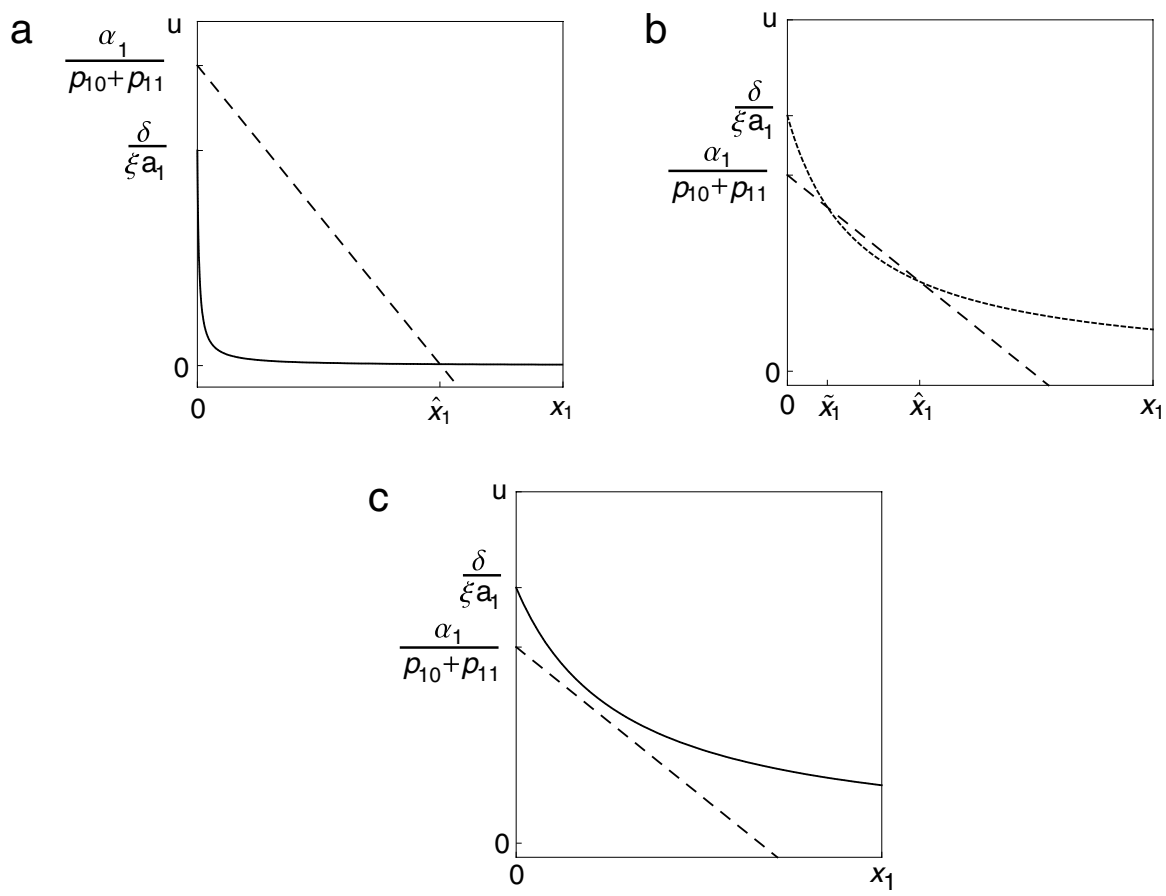

Fig. 1. Curves $\mathcal{C}_{1}$ and $\mathcal{C}_{2}$ for the following conditions: (a) $\delta / \xi a_{1}<\alpha_{1} /\left(p_{10}+p_{11}\right)$, with one cure state; (b) $\delta / \xi a_{1}>\alpha_{1} /\left(p_{10}+p_{11}\right)$, with two cure states; (c) $\delta / \xi a_{1}>\alpha_{1} /\left(p_{10}+p_{11}\right)$, with no cure states.

Eliminating $\hat{y}$ in (12) gives the positive solution

$$
\hat{x}_{1}=\frac{\left(-\xi a_{1}+\xi+d_{1}\right)+\sqrt{\left(\xi a_{1}-\xi-d_{1}\right)^{2}-4\left(\xi+d_{1}\right)\left[\left(p_{10}+p_{11}\right) \delta / \alpha_{1}-\xi a_{1}\right]}}{2\left(\xi+d_{1}\right)},
$$

which provides, together with $\hat{x}_{3}=1$ and (12), the cure solution $\hat{F}$.

Consider the two curves given by $u=\alpha_{1}\left(1-x_{1}\right)\left(a_{1}+x_{1}\right) /\left(p_{10}+p_{11}\right)$ and $u=\left(a_{1}+x_{1}\right) \delta /\left[\left(\xi+d_{1}\right) x_{1}+\xi a_{1}\right]$ shown as $\mathcal{C}_{1}$ and $\mathcal{C}_{2}$ in Fig. 1 in the $x_{1}-u$ plane. As illustrated in Fig. 1, there may be 2, 1, or 0 positive solutions according to the following scheme:

(i) if $\delta / \xi a_{1}<\alpha_{1} /\left(p_{10}+p_{11}\right)$ there is one cure state;

(ii) if $\delta / \xi a_{1}>\alpha_{1} /\left(p_{10}+p_{11}\right)$ there are two or no cure states.

In the case of no cure state, $\hat{x}_{1}>0$ does not exist so that the positive $x_{1}-y$ plane, which is itself invariant, can have no invariant sets. Therefore, $\lim _{t \rightarrow \infty} x_{2}(t)=0$ cannot happen if $x_{1}(t)>0$ holds, i.e. cancer cells cannot be eliminated.

If $\hat{x}_{1}>0$ exists, then $\hat{y}>0$ also exists according to (12). In this case, we are interested in the stability of $\hat{F}\left(\hat{x}_{1}, 0,1, \hat{y}\right)$ with $\hat{x}_{1}>0$ in the $x_{2}$ (cancer) direction.

We now compute the variational matrix of system (3) about the equilibrium $\hat{F}$, which then takes the form:

$$
\hat{\mathbf{M}}=\left(\begin{array}{cccc}
\hat{m}_{11} & \hat{m}_{12} & \hat{m}_{13} & \hat{m}_{14} \\
0 & \hat{m}_{22} & 0 & 0 \\
0 & \beta & -\alpha_{3} & 0 \\
\hat{m}_{41} & \hat{m}_{42} & 0 & \hat{m}_{44}
\end{array}\right) .
$$

Clearly the eigenvalue in the $x_{2}$ (cancer) direction is

$$
\hat{\lambda}_{2}=\hat{m}_{22}=\alpha_{2}-q_{2} \hat{x}_{1}-\left(p_{20}+p_{21}\right) \hat{y} / a_{2} .
$$

If $\hat{m}_{22}<0$, then it may be the case that cancer cells can be eliminated using chemotherapy alone. However, if $\hat{m}_{22}>0$, then it may not be possible to eliminate the cancer cells using only chemotherapy. We are particularly interested in this last case. In order to obtain a firm result of this type, we first derive a condition for the non-existence of any nontrivial closed orbits in the positive $x_{1}-y$ plane when $x_{3}=1$. 
Theorem 2. Consider the system given by

$$
\begin{aligned}
& \dot{x}_{1}=\alpha_{1} x_{1}\left(1-x_{1}\right)-\frac{\left(p_{10}+p_{11}\right) x_{1} y}{a_{1}+x_{1}} \\
& \dot{y}=\delta-\left(\xi+\frac{d_{1} x_{1}}{a_{1}+x_{1}}\right) y .
\end{aligned}
$$

Then, if $a_{1} \geq 1$, there are no nontrivial closed orbit solutions in the positive orthant.

Proof. Write our system as $\dot{x}_{1}=F\left(x_{1}, y\right), \dot{x}_{2}=G\left(x_{1}, y\right)$ and let

$$
\mathscr{B}(x, y)=\left(a_{1}+x_{1}\right) / x_{1} y .
$$

Then we compute

$$
\begin{aligned}
\mathscr{D}\left(x_{1}, y\right) & =\frac{\partial}{\partial x_{1}}\left(\mathscr{B}\left(x_{1}, y\right) F\left(x_{1}, y\right)\right)+\frac{\partial}{\partial y}\left(\mathscr{B}\left(x_{1}, y\right) G\left(x_{1}, y\right)\right) \\
& =\frac{\partial}{\partial x_{1}}\left[\frac{\alpha_{1}\left(1-x_{1}\right)\left(a_{1}+x_{1}\right)}{y}-\left(p_{10}+p_{11}\right)\right]+\frac{\partial}{\partial y}\left[\frac{\delta\left(a_{1}+x_{1}\right)}{x_{1} y}-\frac{\xi\left(a_{1}+x_{1}\right)}{x_{1}}-d_{1}\right] \\
& =\frac{\alpha_{1}\left(1-a_{1}\right)-2 x \alpha_{1}}{y}-\frac{\delta\left(a_{1}+x_{1}\right)}{x_{1} y^{2}}<0
\end{aligned}
$$

whenever $x_{1}>0, y>0, a_{1} \geq 1$. Hence, by Dulac's theorem [33], no closed orbit solutions exist in the region $x>0, y$ $>0$.

The above result can be combined with ideas of persistence theory [34] and with the fact that $x_{2}(t) \rightarrow 0$ then $x_{3} \rightarrow 1$, leading to the following

Theorem 3. Let $a_{1} \geq 1$ and $\hat{m}_{22}>0$ (if it exists) be given by (14). Then system (9) exhibits persistence.

An interpretation of the above result is that, if no cure state exists or if one exists but is unstable in the $x_{2}$-direction, then CCs cannot be eliminated by chemotherapy alone, provided $a_{1} \geq 1$. That means that CA kills CCs but does not eliminate the tumour as it is observed for drug-resistant tumours [26]. Note that the condition $a_{1} \geq 1$ is a necessary condition to guarantee that the system evolves to the interior equilibrium with NCs and CCs: $a_{1} \geq 1$ corresponds to an actual situation for which the saturation of the killing action of CA on NCs is not negligible. This is associated with a cytotoxic effect of CA.

\section{Analytical results in the full model}

We now come back to analyse in part system (3). We are once more interested in the case where there is an equilibrium of the form $x_{2}=0, x_{1}>0$. We denote such an equilibrium as $E^{*}\left(x_{1}^{*}, 0, x_{3}^{*}, y^{*}, w^{*}\right)$, obtained by solving the algebraic system

$$
\left\{\begin{array}{l}
\alpha_{1}\left(1-x_{1}\right)-\left(p_{10}+p_{11} x_{3}+p_{12} w\right) y /\left(a_{1}+x_{1}\right)=0 \\
\alpha_{3}\left(1-x_{3}\right)-p_{3} w /\left(a_{3}+x_{3}\right)=0 \\
\delta-\left[\xi+d_{1} x_{1} /\left(a_{1}+x_{1}\right)\right] y=0 \\
\phi-\left[\eta+d_{3} x_{3}\left(a_{3}+x_{3}\right)\right] w=0 .
\end{array}\right.
$$

As before, we do not consider the case where $x_{3}^{*}=0$. Then we wish to solve the system of two equations:

$$
\begin{aligned}
& w=\frac{\alpha_{3}\left(1-x_{3}\right)\left(a_{3}+x_{3}\right)}{p_{3}} \\
& w=\frac{\left(a_{3}+x_{3}\right) \phi}{\left(\eta+d_{3}\right) x_{3}+\eta a_{3}} .
\end{aligned}
$$

Eliminating $w$ in (18) and writing out the resulting quadratic equation we obtain

$$
\alpha_{3}\left(\eta+d_{3}\right) x_{3}^{2}+\alpha_{3}\left(\eta a_{3}-\eta-d_{3}\right) x_{3}+\left(p_{3} \phi-\alpha_{3} \eta a_{3}\right)=0 .
$$

Note that if $p_{3} \phi<\alpha_{3} \eta a_{3}$ then, by Descarte's rule of signs, there must exist exactly one positive root of this quadratic equation given by

$$
x_{3}^{*}=\frac{\left(-\eta a_{3}+\eta+d_{3}\right)+\sqrt{\left(\eta a_{3}-\eta-d_{3}\right)^{2}-4\left(\eta+d_{3}\right)\left[\phi\left(p_{3}\right) / \alpha_{3}-\eta a_{3}\right]}}{2\left(\eta+d_{3}\right)} .
$$


Now, having found $w^{*}$ and $x_{3}^{*}$ given by (18) and (19) respectively, we solve

$$
\left\{\begin{array}{l}
\alpha_{1}\left(1-x_{1}\right)-\left(p_{10}+p_{11} x_{3}^{*}+p_{12} w^{*}\right) y /\left(a_{1}+x_{1}\right)=0 \\
\delta-\left[\xi+d_{1} x_{1} /\left(a_{1}+x_{1}\right)\right] y=0
\end{array}\right.
$$

or

$$
\begin{aligned}
y^{*} & =\frac{\alpha_{1}\left(1-x_{1}^{*}\right)\left(a_{1}+x_{1}^{*}\right)}{\left(p_{10}+p_{11} x_{3}^{*}+p_{12} w^{*}\right)} \\
y^{*} & =\frac{\left(a_{1}+x_{1}^{*}\right) \delta}{\left(\xi+d_{1}\right) x_{1}^{*}+\eta a_{1}} .
\end{aligned}
$$

As $x_{1}^{*}$ increases from 0 to 1 , in the first equation of the above equations, $y^{*}$ goes from $\alpha_{1} a_{1} /\left(p_{10}+p_{11} x_{1}^{*}+p_{12} w^{*}\right)$ to 0 (strictly decreasing after some value of $x_{1}^{*}$ ). In the second equation for $y^{*}$, as $x_{1}^{*}$ goes from 0 to $1, y^{*}$ decreases from $\delta / \xi$ to $\left(a_{1}+1\right) \delta /\left[\left(a_{1}+1\right) \xi+d_{1}\right]$. Hence, if $\delta / \xi<\alpha_{1} a_{1} /\left(p_{10}+p_{11} x_{3}^{*}+p_{12} w^{*}\right), x_{1}^{*}>0$ always exists and so also must $y^{*}$. In this case, $x_{1}^{*}$ is given by

$$
x_{1}^{*}=\frac{\left(\xi+d_{1}-\xi a_{1}\right)+\left[\left(\xi+d_{1}-\xi a_{1}\right)\right]^{2}-4\left(\xi+d_{1}\right)\left[p_{1}\left(x_{3}^{*}, w^{*}\right) \delta / \alpha_{1}-\xi a_{1}\right]^{1 / 2}}{2\left(\xi+d_{1}\right)}
$$

with $p_{1}\left(x_{3}^{*}, w^{*}\right)=p_{10}+p_{11} x_{3}^{*}+p_{12} w^{*}$. This provides, together with (18) and (19), the cure solution $E^{*}$.

To determine the stability of $E^{*}$, one may compute the variational matrix of system (3) about $E^{*}$ given by

$$
\mathbf{M}^{*}=\left(\begin{array}{ccccc}
m_{11}^{*} & m_{12}^{*} & m_{13}^{*} & m_{14}^{*} & m_{15}^{*} \\
0 & m_{22}^{*} & 0 & 0 & 0 \\
0 & \beta & m_{33}^{*} & 0 & m_{35}^{*} \\
m_{41}^{*} & m_{42}^{*} & 0 & m_{44}^{*} & 0 \\
0 & 0 & m_{53}^{*} & 0 & m_{55}^{*}
\end{array}\right) .
$$

However, to simply get the local stability of $E^{*}$ in the $x_{2}$-direction, it is sufficient to note that one of the eigenvalues $\left(\lambda_{2}^{*}=m_{22}^{*}\right)$ must satisfy the following inequality

$$
\lambda_{2}^{*}=m_{22}^{*}=\alpha_{2}-q_{2} x_{1}^{*}-\left(p_{20}+p_{21} x_{3}^{*}+p_{22} w^{*}\right) y^{*} / a_{2}<0 .
$$

There is now the possibility of a reversal of outcome. Suppose that $\hat{\lambda}_{2}>0$ (see Eq. (14)), so that the cure state of the no anti-angiogenic model is not attainable. However, if $\lambda_{2}^{*}<0$, the system can be brought by whatever means sufficiently close to $E^{*}$, so that $\lim _{t \rightarrow \infty} x_{2}(t)=0$ may be achieved and the outcome is reversed. Therefore we obtain the following.

Remark 2. The region of parameter space where a reversal of outcome occurs is such that $\hat{\lambda}_{2}$, given by (14), is positive and $\lambda_{2}^{*}$, given by (23), is negative.

These analytical results of Sections 3 and 4 are illustrated by the numerical examples in the next section.

\section{Numerical results}

In order to perform numerical integrations of the model, it is necessary to restrict the parameter space taking into account realistic values. As in the model introduced in [9], besides the conditions (5), we also impose some restrictions on the parameters of system (1) based on the following inequalities.

(a) CCs grow at a faster rate than NCs: $\alpha_{2}>\alpha_{1}$. For some human tumours, it is observed that the proliferation rate $\alpha_{2}$ is about $10^{-2}$ day $^{-1}[35]$;

(b) The chemical agent must be considerably more effective in killing CCs than NCs: $P_{20} \gg P_{10}$ [36]; analogously $d_{2}>d_{1}$.

(c) The competition effect on NCs is more effective than on CCs $\left(Q_{1}>Q_{2}\right)$.

(d) The cell death rate of ECs is smaller than its proliferation rate due to the tumour $\left(\alpha_{3}<B\right)$ [4]; it is also smaller than the proliferation rate of NCs $\left(\alpha_{3}<\alpha_{1}\right)$ [37].

(e) The saturation parameters $A_{1}$ and $A_{2}$ are similar to the inverse values of carrying capacities of NCs and CCs respectively.

(f) The assistances of ECs and AA to the CA are smaller than their own action $\left(P_{1 i} \leq P_{10}\right), i=1,2$.

(g) The used numerical values of infusion rates of CA $(\delta)$ and AA $(\phi)$ are based on their actual doses per tumour mass, e.g., $170 \mathrm{mg} /(\mathrm{kg})($ day) of cyclophosphamide and $12.5 \mathrm{mg} /(\mathrm{kg})($ day) of TNP-470 respectively [26]. The doses are almost continuous (very short periods between the infusions) since they are administered in a 6 day dosage for 7 cycles. As stated in [26], the relation between the chemotherapeutic and anti-angiogenic doses is such that $\delta=14 \phi$.

(h) Analogously the relation between the washout of $C A(\xi)$ and AA $(\eta)$ is based on the relation between the half-life of CA (cyclophosphamide) [38] and AA (TNP-40) [39] in mice: $\eta=7.5 \xi$. 
Table 1

Parameters of the model.

\begin{tabular}{|c|c|c|c|}
\hline Description & Parameter & Value & Comment/reference \\
\hline NCs proliferation rate & $\alpha_{1}$ & 0.0068 day $^{-1}$ & $\alpha_{1}<\alpha_{2}$ \\
\hline CCs proliferation rate & $\alpha_{2}$ & 0.01 day $^{-1}$ & Experimental data [35] \\
\hline ECs proliferation rate & $\alpha_{3}$ & 0.002 day $^{-1}$ & $\alpha_{3}<\alpha_{1}[37]$ \\
\hline Carrying capacity of NCs & $K_{1}$ & 200 & Normalized factor for $X_{1}$ \\
\hline Carrying capacity of CCs & $K_{2}$ & 195 & Normalized factor for $X_{2}$ \\
\hline Carrying capacity of ECs & $K_{3}$ & 210 & Normalized factor for $X_{3}$ \\
\hline Competition coefficient for NCs & $Q_{1}$ & $3.6 \times 10^{-5} \mathrm{day}^{-1}$ & Cancer hypothesis \\
\hline Competition coefficient for CCs & $Q_{2}$ & $3.6 \times 10^{-6}$ day $^{-1}$ & $Q_{2}<Q_{1}$ \\
\hline Proportion of ECs due to angiogenesis & $\Gamma$ & 0.15 & $\Gamma<1$ \\
\hline Rate of creation of CCs due to ECs & $B$ & 0.004 day $^{-1}$ & $B>\alpha_{3}[4]$ \\
\hline Saturation rate of the agent on NCs & $A_{1}$ & 220 & $A_{1}>K_{1}$ (Theorem 2$)$ \\
\hline Saturation rate of the agent on CCs & $A_{2}$ & 901 & $A_{2} \approx K_{2}$ \\
\hline Saturation rate of the agent on ECs & $A_{3}$ & 980 & $A_{3} \approx K_{3}$ \\
\hline Rate at which CA combines with NCs & $d_{1}$ & 0.0002 day $^{-1}$ & Estimated value \\
\hline Rate at which CA combines with CCs & $d_{2}$ & 0.032 day $^{-1}$ & $d_{2} \gg d_{1}$ \\
\hline Rate at which AA combines with ECs & $d_{3}$ & 0.032 day $^{-1}$ & $d_{3} \geq d_{2}[18]$ \\
\hline Killing rate of CA on NCs & $P_{10}$ & $2.4 \times 10^{-5}$ day $^{-1}$ & Estimated value \\
\hline Killing rate of CA on CCs & $P_{20}$ & $4.0 \times 10$ day $^{-1}$ & $P_{20} \gg P_{10}[36]$ \\
\hline Killing rate of AA on ECs & $P_{3}$ & $3.6 \times 10^{2}$ day $^{-1}$ & $P_{3} \geq P_{20}$ \\
\hline Rate of ECs assistance on CA for NCs & $P_{11}$ & $4.0 \times 10^{-8} \mathrm{day}^{-1}$ & $P_{11}<P_{10}$ \\
\hline Rate of ECs assistance on CA for CCs & $P_{21}$ & $4.0 \times 10^{-3}$ day $^{-1}$ & $P_{21}>P_{11}$ \\
\hline Rate of AA assistance on CA for NCs & $P_{12}$ & $2.0 \times 10^{-5}$ day $^{-1}$ & $P_{12}<P_{10}$ \\
\hline Rate of AA assistance on CA for CCs & $P_{22}$ & $3.8 \times 10^{3}$ day $^{-1}$ & $P_{22}>P_{12}$ \\
\hline Chemical infusion rate & $\delta$ & $3.3 \times 10^{-3} \mathrm{day}^{-1}$ & Almost continuous [26] \\
\hline Chemical washout rate & $\xi$ & $0.01813 \mathrm{day}^{-1}$ & Half-life of CA [38] \\
\hline Anti-angiogenic infusion rate & $\phi$ & $2.4 \times 10^{-4} \mathrm{day}^{-1}$ & $\delta=14 \phi[26]$ \\
\hline Anti-angiogenic washout rate & $\eta$ & 0.136 day $^{-1}$ & $\eta=7.5 \xi[39]$ \\
\hline
\end{tabular}

Table 2

Parameters of the normalized model.

\begin{tabular}{lll}
\hline Parameter & Normalized relation & Value \\
\hline$\alpha_{1}$ & - & $0.0068 \mathrm{day}^{-1}$ \\
$\alpha_{2}$ & - & $0.01 \mathrm{day}^{-1}$ \\
$\alpha_{3}$ & - & $0.002 \mathrm{day}^{-1}$ \\
$q_{1}$ & $Q_{1} K_{2}$ & $0.00702 \mathrm{day}^{-1}$ \\
$q_{2}$ & $Q_{2} K_{1}$ & $0.00072 \mathrm{day}^{-1}$ \\
$\gamma$ & $\Gamma K_{3} / K_{2}$ & 0.1615 \\
$\beta$ & $B K_{2} / K_{3}$ & $0.00371 \mathrm{day}^{-1}$ \\
$a_{1}$ & $A_{1} / K_{1}$ & 1.10 \\
$a_{2}$ & $A_{2} / K_{2}$ & 4.6205 \\
$a_{3}$ & $A_{3} / K_{3}$ & 4.6666 \\
$d_{1}$ & - & $0.0002 \mathrm{day}^{-1}$ \\
$d_{2}$ & - & $0.032 \mathrm{day}^{-1}$ \\
$d_{3}$ & - & $0.032 \mathrm{day}^{-1}$ \\
$p_{10}$ & $P_{10} / K_{1}$ & $1.2 \times 10^{-7}$ day $^{-1}$ \\
$p_{20}$ & $P_{20} / K_{2}$ & 0.2051 day $^{-1}$ \\
$p_{3}$ & $P_{3} / K_{3}$ & 1.7143 day $^{-1}$ \\
$p_{11}$ & $P_{11} K_{3} / K_{1}$ & $4.2 \times 10^{-8}$ day $^{-1}$ \\
$p_{12}$ & $P_{12} / K_{1}$ & $1.0 \times 10^{-7}$ day $^{-1}$ \\
$p_{21}$ & $P_{21} K_{3} / K_{2}$ & 0.00431 day $^{-1}$ \\
$p_{22}$ & $P_{22} / K_{2}$ & 19.4872 day $^{-1}$ \\
$\delta$ & - & $3.3 \times 10^{-3}$ day $^{-1}$ \\
$\xi$ & - & 0.01813 day $^{-1}$ \\
$\phi$ & - & $2.4 \times 10^{-4}$ day $^{-1}$ \\
$\eta$ & - & 0.136 day $^{-1}$ \\
\hline & &
\end{tabular}

In Table 1 we present the parameter values for the original model (1) with some comments and/or references. The estimated values for $d_{1}$ and $P_{10}$ are related to the existence and unstable character of the cure state in the no anti-angiogenic model. Table 2 shows the normalized parameter values adopted on the numerical integrations, according to their relations to the parameters in Table 1.

In Figs. 2 and 3 we present numerical examples of the main result of our paper. In Fig. 2, an example of time evolution of the no anti-angiogenic sub-model (9) under the conditions of Theorems 2 and 3 is shown. It simulates a situation in which the CCs are not eliminated. On the other hand, Fig. 3 shows a positive scenario described by Remark 2 for which the full 


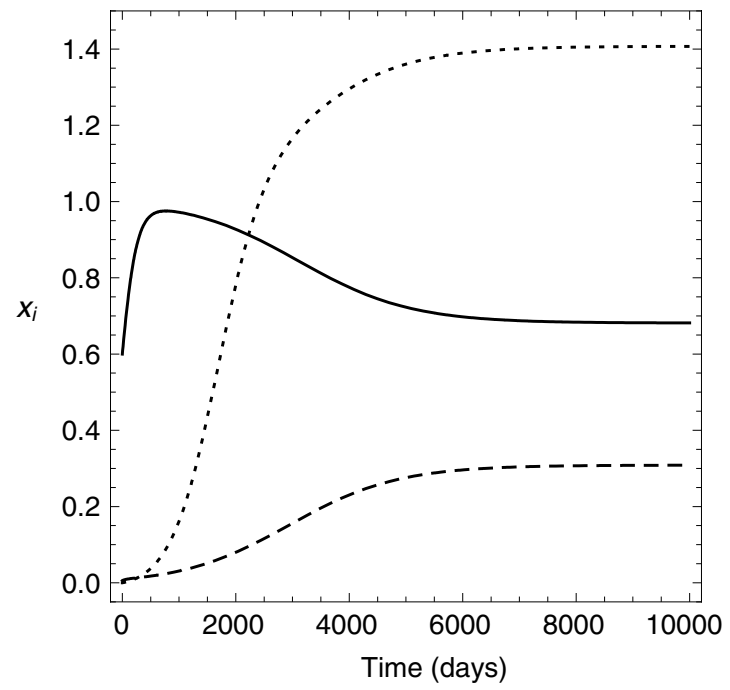

Fig. 2. Time evolution of the no anti-angiogenic sub-model using parameter values in Table 2. Thick line-NCs; dashed line-CCs; dotted line-ECs. The initial conditions are: $x_{1}(0)=0.6, x_{2}(0)=0.006 ; x_{3}(0)=y(0)=w(0)=0$. The system evolves to the internal steady state: $x_{1}=0.681396, x_{2}=0.308617$, $x_{3}=1.40727, y=0.163258$.

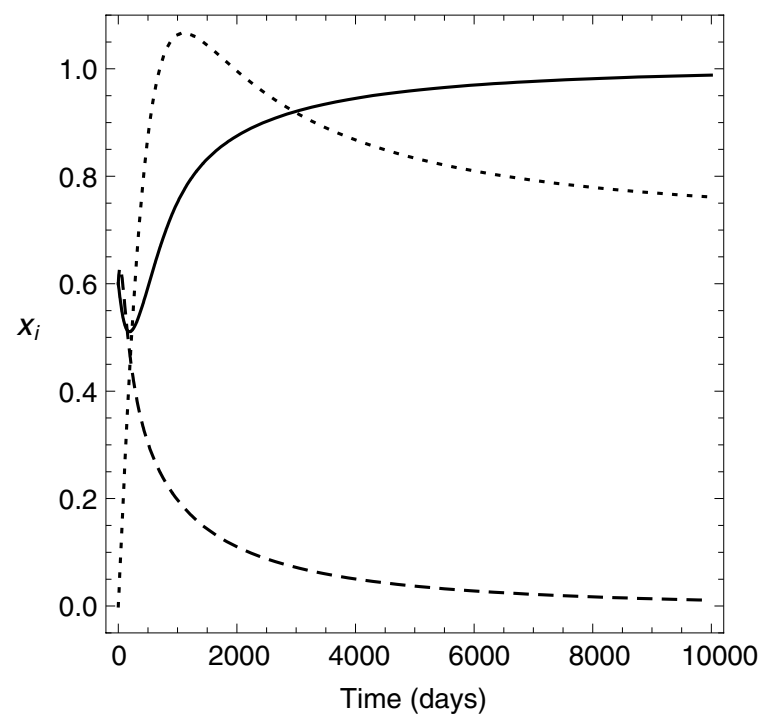

Fig. 3. Time evolution of the full model using parameter values in Table 2. Thick line-NCs; dashed line-CCs; dotted line-ECs. The initial conditions are: $x_{1}(0)=0.6, x_{2}(0)=0.6 ; x_{3}(0)=y(0)=w(0)=0$. The system evolves to the cure steady state: $x_{1}=0.999998, x_{2}=0.0, x_{3}=0.728255, y=$ $0.181034, w=0.00171038$.

model (3) evolves to a cure state. Note that this simulates a reversal of outcome, i.e., the co-administration leads to the elimination of CCs, not just to a reduction of tumour size.

The influence of the CA infusion rates, which are easily controlled in real cases and may assume quite different values, is illustrated by the bifurcation diagrams in Figs. 4 and 5, There it is possible to follow the evolution from the worst to best scenarios: CCs win the fight with NCs (cancer state), the reduction of tumour size (internal state), and the elimination of CCs (cure state). These diagram bifurcations were obtained numerically [40]. In all cases, there are transcritical bifurcations both from the cancer to the internal state, and from the internal to the cure state. They provide a comparing view of the transitions between the steady states - cure state $\left(x_{2}=0\right)$, internal state and cancer state $\left(x_{1}=0\right)$ - of the no anti-angiogenic submodel and the full model, shown in Figs. 4 and 5 respectively.

For the full model, the cure state is observed for a larger range of values of $\delta$. In case the system evolves to an internal state, it is also easy to see in Fig. 5(b) that, for a fixed value of $\delta$, the CCs population is reduced more effectively for the full model than for the no anti-angiogenic model. Note that the relative deviation of the value of $\delta$ for the cure transition between the no anti-angiogenic sub-model and the full model is about $15 \%=|0.0032-0.0037| 100 / 0.0032$. 
a

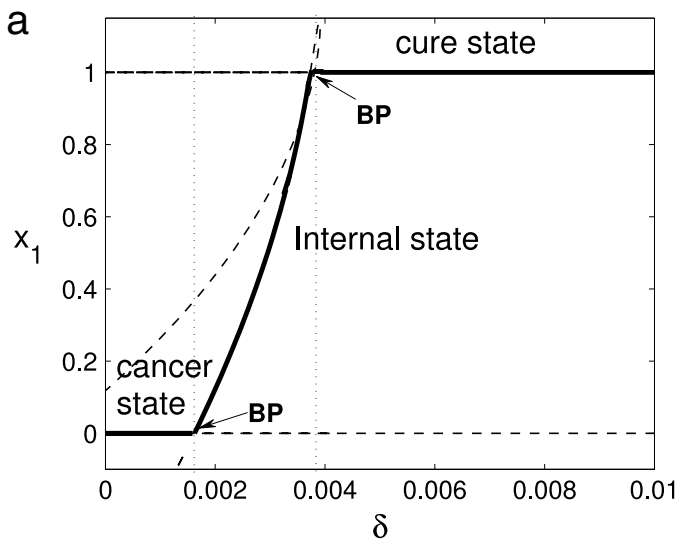

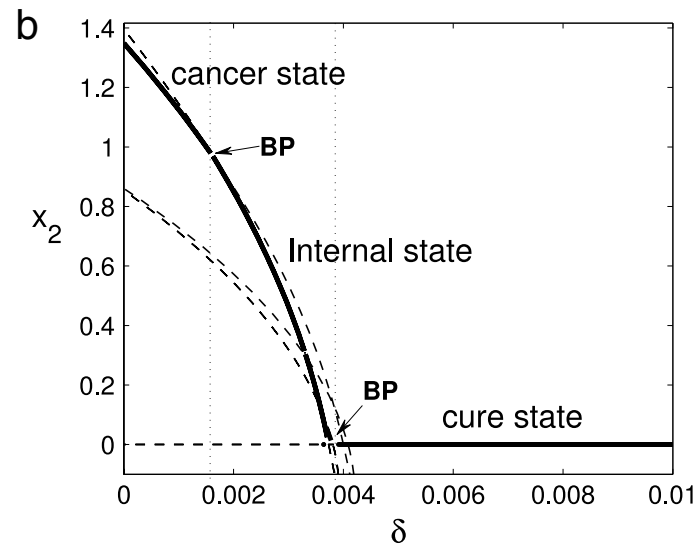

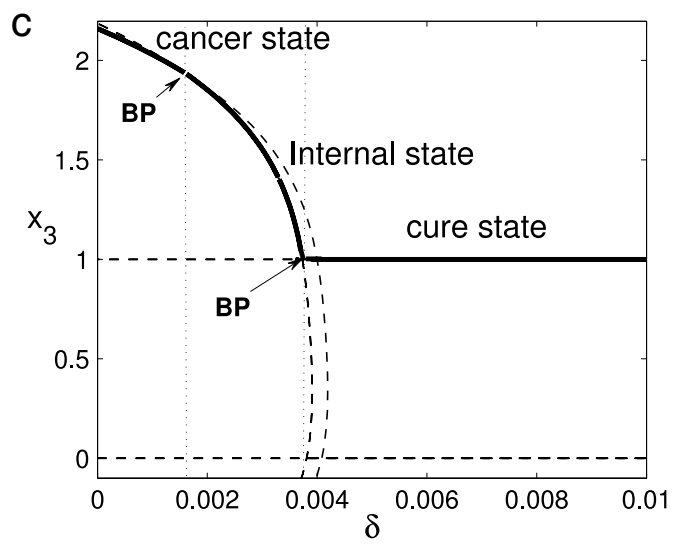

Fig. 4. Bifurcation diagrams of the no anti-angiogenic sub-model for NC (a), CC (b) and EC (c) densities in terms of the chemotherapy infusion rate $\delta$. Parameter values of the sub-model are taken from Table 2, except for $\delta$ which varies from 0 to 0.01 day $^{-1}$. Two transcritical bifurcations (BP) are indicated: from the cancer to the internal state at $\delta=0.001623$ day $^{-1}$; from the internal to the cure state at $\delta=0.003732$ day $^{-1}$.

Since the parameter $p_{22}$ affects the full model but not the no anti-angiogenic model, we show in Fig. 6 a two-dimensional bifurcation diagram for the full model that makes evident how the cure region of the parameter space may be increased. This points out a very important issue: increasing the AA efficiency in vascularization normalizing, represented by the parameter $p_{22}$ in our model, is a good strategy to reach a cure state by reducing the chemical infusion rate $\delta$.

\section{Discussion and concluding remarks}

In this paper we present a model of cancer treatment including anti-angiogenic drugs which aid chemotherapeutic drugs for chemo-resistant tumours. We take into account the competition between NCs and CCs as well as the growth of the ECs population associated with the angiogenic process, which helps the tumour growth. We prove that the full model is bounded and dissipative (Theorem 1). We also impose the main assumption of a 'cancer hypothesis' in the no therapy case, i.e., the system evolves to a cancer state [9], provided conditions (5) hold. Under this hypothesis, we prove that anti-angiogenic therapy alone is not able to drive the system to a cure state. This conclusion, stated by Remark 1, was already pointed out in $[22,25]$.

Theorems 2 and 3 determine the conditions for which the no anti-angiogenic model does not lead to the cure state, which is also evident in Fig. 2. However, including anti-angiogenic drugs may lead to a reversal of outcome as pointed out in Remark 2, and illustrated in Fig. 3. This behaviour has been observed for the co-administration of both chemical and anti-angiogenic drugs for drug-resistant tumours in mice [26]. The enhancement of the combined action of CA and AA is evident when we compare the bifurcation diagrams shown in Figs. 4 and 5. The bifurcation diagram for the full model (see Fig. 5) also shows the situation for which the tumour is reduced more effectively for the combined therapies than for the chemotherapy alone as was observed for some tumour types [22].

Finally, based on the two-parameter diagram bifurcation of the full model (see Fig. 6) it is possible to reduce the chemotherapy infusion $(\delta)$ and to increase the anti-angiogenic assistance effect to the conventional chemotherapy action $\left(p_{22}\right)$ in order to get a better response in the treatment of cancer. For actual situations, to increase $p_{22}$ means to use antiangiogenic drugs that are more effective in normalizing the vascularization; simultaneously, decreasing the chemotherapy infusion corresponds to reducing the cytotoxic effect on NCs. 

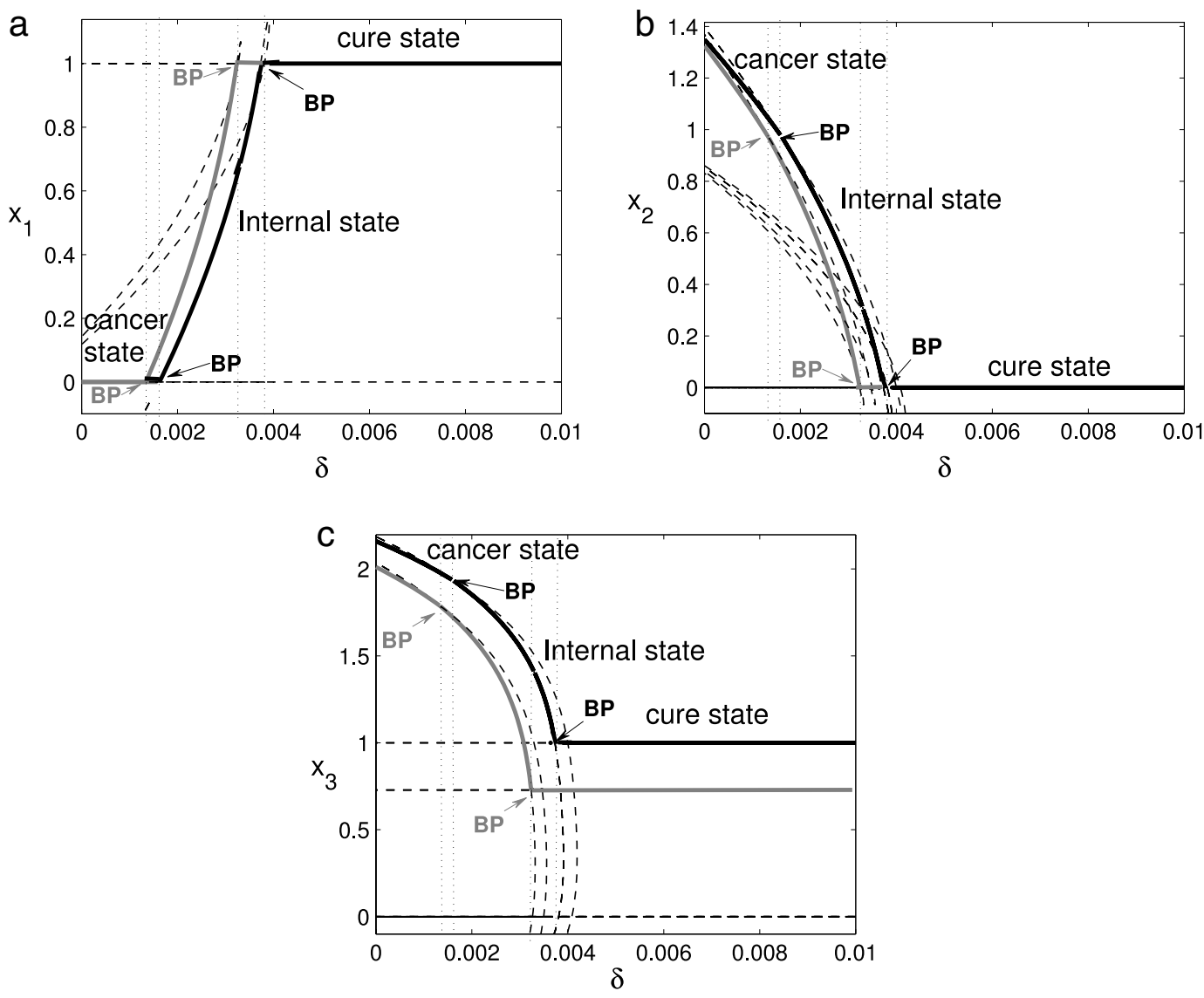

Fig. 5. Bifurcation diagrams of the full model for NC (a), CC (b) and EC (c) densities in terms of the chemotherapy infusion rate $\delta$. Parameter values are taken from Table 2, except for $\delta$ which varies from 0 to 0.01 . Grey and black lines correspond, respectively, to the full model and the no anti-angiogenic sub-model. Two transcritical bifurcations (BP) are indicated: from the cancer to the internal state at $\delta=0.001341$ day $^{-1}$; from the internal to the cure state at $\delta=0.003253 \mathrm{day}^{-1}$. This value of $\delta$ is almost $15 \%$ smaller than the corresponding cure transition value in the no anti-angiogenic sub-model.

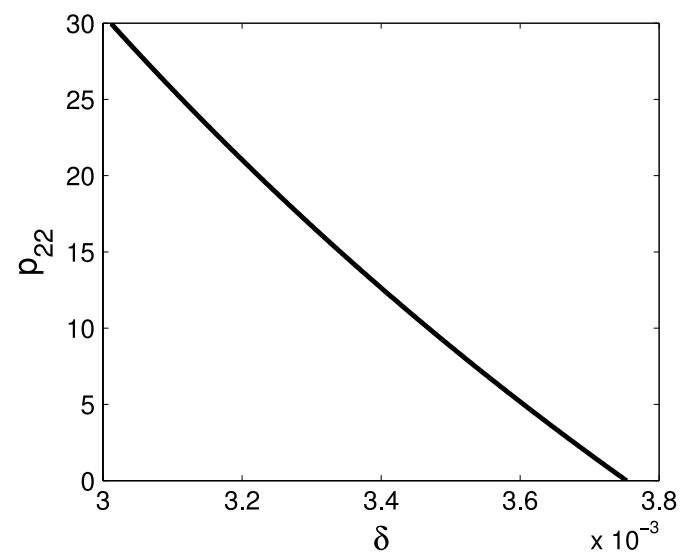

Fig. 6. Two parameters bifurcation diagram $p_{22} \times \delta$ for the full model in the parameter region where the bifurcation from the internal to the cure state is observed. Parameter values are taken from Table 2, except for $\delta$ and $p_{22}$, that vary, respectively, from 0 to 0.0038 day ${ }^{-1}$, and from 0 to 30 day ${ }^{-1}$.

Summarizing, the results of our models show relevant clinical features of the therapies as follows.

(a) It is not possible to eliminate the cancer cells by anti-angiogenic therapy alone.

(b) If a drug-resistant tumour cannot be eliminated by chemotherapy alone, a reversal of outcome may be attained by combining simultaneously chemotherapy and anti-angiogenic therapies, as was pointed out by some experiments with mice [26]. 
(c) The co-administration of chemical and anti-angiogenic agents is able to promote a larger reduction of the tumour than the chemotherapy alone.

Initially, Folkman [17] argued that anti-angiogenic agent may lead to cure, although nowadays it is generally assumed that this is not true. Here we lend credence to this assumption in our models. In addition we indicate that this scenario may be attained using AA that are efficient in helping the delivery of CA due to the normalization of the vasculature.

Moreover, the continuous treatment may be thought as a limiting case of small intervals between infusions, as in Ref. [26]. In some sense our analysis of combining continuous infusions of CA and AA leads to a discussion about the elimination of tumours with smaller doses of CA, which are applied simultaneously with AA. This strategy also has the effect of reducing the cytotoxic effect on NCs.

While in this work we analyse the response of drug-resistant tumours to the co-administration of CA and AA, the investigation on different schedules of chemotherapy for chemo-sensitive tumours [41] has been pushed forward by one of us and other collaborators. In such a case, the anti-angiogenic side effect of chemotherapy is explicitly taken into account. Our aim is to compare the conventional (maximal tolerated dose) schedule of chemotherapy with metronomic chemotherapy schedule with small intervals between infusions.

In future research, we intend to consider the case where there is a time delay between the tumour growth and the neovascularization of the tumour, which is observed in some experiments with mice [42]. A change in vascularization does not immediately affect the tumour growth. Some events take place in the time interval limited by the instant in which angiogenic factors are released from the solid tumour to the instant when vascularization takes place [14].

\section{Acknowledgments}

The authors wish to thank an anonymous referee for several suggestions which have led to an improved manuscript. This work has been partially supported by the Brazilian agencies CNPq (INCT-Complex Systems, INCT- CITECS), FAPESB/PRONEX, and the Spanish Agency AECI.

\section{References}

[1] T. Alarcon, M.R. Owen, H.M. Byrne, P.K. Maini, Multiscale modelling of tumour growth and therapy: the influence of vessel normalisation on chemotherapy, Comput. Math. Methods Med. 7 (2006) 85-119.

[2] B. Ribba, T. Colin, S. Schnell, A multiscale mathematical model of cancer, and its use in analyzing irradiation therapies, Theor. Biol. Med. Model. 3 (2006) $1-19$.

[3] A.R.A. Anderson, M.A. Chaplain, Mathematical models of tumour-induced angiogenesis, Bull. Math. Biol. 60 (1998) 857-899.

[4] R.K. Sachs, L.R. Hlatky, P. Hanfeldt, Simple ODE models of tumour growth and anti-angiogenic or radiation treatment, Math. Comput. Modelling 33 (2001) 1297-1305.

[5] W. Liu, H.I. Freedman, A mathematical model of vascular tumour treatment by chemotherapy, Math. Comput. Modelling 42 (2005) $1089-1112$.

[6] P. Magni, M. Simeoni, I. Poggesi, M. Roccheti, G. de Nicolao, A mathematical model to study the effects of drugs administration on tumour growth dynamics, Math. Biosci. 220 (2006) 127-151.

[7] A. d'Onofrio, A. Gandolfi, Tumour eradication by anti-angiogenic therapy: analysis and extensions of the model by Hahnfeldt et al. (1999), Math. Biosci. 191 (2004) 159-184.

[8] R.A. Gatenby, Application of competition theory to tumour growth: implications for tumour biology and treatment, Eur. J. Cancer 32A (1996) $722-726$.

[9] S.T.R. Pinho, H.I. Freedman, F. Nani, A chemotherapy model for the treatment of cancer with metastasis, Math. Comput. Modelling 36 (2002) 773-803.

[10] A.P. Gillio, C. Gasparetto, J. Laver, M. Abboud, M.A. Bonilla, M.B. Garnick, R.J. O'Reilly, Effects of interleukin-3 on hematopoietic recovery after 5-fluorouracil or cyclophosphamide treatment of cynomolgus primates, J. Clin. Investig. 85 (1990) 1560-1565.

[11] C.M. Evans, The Metastatic Cell, Behaviour and Biochemistry, Chapman and Hall, London, 1991.

[12] G.V. Sherbet, The Biology of Tumour Malignancy, Academic Press, London, 1982.

[13] F. Bussolino, M. Arese, E. Audero, E. Giraudo, S. Marchió, S. Mitola, L. Primo, G. Serini, Biological aspects of tumour angiogenesis, in: L. Preziosi (Ed.), Cancer Modelling and Simulation, Chapman \& Hall, CRC, London, 2003, pp. 1-22.

[14] S.A. Maggelakis, The effects of tumour angiogenesis factor (TAF) and tumour inhibitor factors (TIFs) on tumour vascularization: a mathematical model, Math. Comput. Modelling 23 (1996) 121-133.

[15] A. Bikfalvi, Significance of angiogenesis in tumour progression and metastasis, Eur. J. Cancer 31A (1995) 1101-1104.

[16] P. Hahnfeldt, D. Panigrahy, J. Folkman, L. Hlatky, Tumour development under angiogenic signaling: a dynamical theory of tumour growth, treatment response, and postvascular dormancy, Cancer Res. 59 (1999) 4770-4775.

[17] J. Folkman, Tumour angiogenesis: therapeutic implications, N. Engl. J. Med. 285 (1971) 1182-1186.

[18] D. Hanahan, J. Folkman, Patterns and emerging mechanisms of the angiogenic switch during tumourigenesis, Cell 86 (1996) $353-364$.

[19] W.C. Beecken, A. Fernandes, A.M. Joussen, E. Achilles, E. Flynn, K. Lo, S.D. Gilies, K. Javaherian, J. Folkman, Y. Shing, Effect of anti-angiogenic therapy on slowly growing, poorly vascularized tumours in mice, J. Nat. Cancer 93 (2001) 382-387.

[20] R.S. Kerbel, F. Bertolini, S. Man, D.A. Hicklin, U. Emmenegger, Y. Shaked, Anti-angiogenic drugs as broadly effective chemosensitizing agents, in: N. Ferrara (Ed.), Angiogenesis: From Basic Science to Clinical Applications, Taylor \& Francis, New York, 2007, pp. 181-198.

[21] R.K. Jain, Normalizing tumour vasculature with anti-angiogenic therapy: a new paradigm for combination therapy, Nat. Med. 7 (2001) $987-989$.

[22] H. Morioka, L. Weissbach, T. Vogel, G.P. Nielsen, G.T. Faircloth, L. Shao, F.J. Hornicek, Anti-angiogenic treatment combined with chemotherapy produces chondrosarcoma necrosis, Clin. Cancer Res. 9 (2003) 1211-1217.

[23] R.S. Kerbel, G. Klement, K.I. Pritchard, B. Kamen, Editorial: continuous low-dose anti-angiogenic/metronomic chemotherapy: from the research laboratory into the oncology clinic, Ann. Oncol. 13 (2002) 12-15.

[24] A. d'Onofrio, U. Ledzewicz, H. Maurer, H. Schattler, On optimal delivery of combination therapy for tumours, Math. Biosci. 222 (2009) 13-26.

[25] S.S. Lakka, J.S. Rao, Anti-angiogenic therapy in brain tumours, Expert Rev. Neurother. 8 (2008) 1457-1473.

[26] T. Browder, C.E. Butterfield, B.M. Kräling, B. Shi, B. Marshall, M.S. OReilly, J. Folkman, Anti-angiogenic scheduling of chemotherapy improves efficacy against experimental drug-resistant cancer, Cancer Res. 60 (2001) 1878-1886.

[27] P.J. O'Dwyer, The present and future of angiogenesis-directed treatments of colorectal cancer, Oncologist 11 (2006) 992-998.

[28] J.D. Murray, Mathematical Biology, Springer Verlag, Berlin, 1993.

[29] E.A. Coddigton, N. Levinson, Theory of Ordinary Differential Equations, McGraw Hill, New York, 1955.

[30] F.K. Nani, H.I. Freedman, A mathematical model of cancer treatment by immunotherapy, Math. Biosci. 163 (2000) 159-199. 
[31] H.I. Freedman, Deterministic Mathematical Models in Population Ecology, Marcel Dekker, New York, 1980.

[32] V. Lakshmikantham, S. Leela, Differential and Integral Inequalities: Theory and Applications, Academic Press, New York, 1969.

[33] A.A. Andronov, E.A. Leontovich, I.I. Jordan, A.G. Maier, Qualitative Theory of Second-Order Dynamic Systems, John Wiley \& Sons, New York, 1973.

[34] H.I. Freedman, P. Waltman, Persistence in models of three interacting predator-prey populations, Math. Biosci. 68 (1984) $213-231$.

[35] J.S. Spratt, J.A. Meyer, Rates of growth of human neoplasms: part II, J. Surg. Oncol. 61 (1996) 68-83.

[36] R.T. Silver, R.D. Lauper, C.I. Jarowski, A Synopsis of Cancer Chemotherapy, Yorke Medical Books, New York, 1987.

[37] B. Alberts, A. Johnson, J. Lewis, M. Raff, K. Roberts, P. Walter, Molecular Biology of the Cell, Taylor \& Francis Books, London, 2002.

[38] R. Said, M. Abdel-Rehim, B. Sadeghia, S. Al-Hashemi, Z. Hassana, M. Hassan, Cyclophosphamide pharmacokinetics in mice: a comparison between retro orbital sampling versus serial tail vein bleeding, Open Pharmacol. J. 1 (2007) 30-35.

[39] S. Shusterman, S.A. Grupp, R. Barr, D. Carpentieri, H. Zhao, J.M. Maris, The angiogenesis inhibitor TNP-470 effectively inhibits human neuroblastoma xenograft growth especially in the setting of subclinical disease, Clin. Cancer Res. 7 (2001) 977-984.

[40] E.J. Doedel, A.R. Champneys, T.F. Fairgrieve, Y.A. Kuznetsov, B. Sandstede, X. Wang, Auto 97: continuation and bifurcation software for ordinary differential equations, Technical Report, Concordia University, Montreal, 1997.

[41] D.S. Rodrigues, P.F.A. Mancera, S.T.R. Pinho, A simple mathematical model for vascular tumours under different chemotherapy schedules, B. Math. Biol. (submitted for publication), 2012.

[42] L. Arakelian, Y. Merbl, P. Daugulis, Y. Ginosar, V. Vainstein, V. Selitser, Y. Kogan, H. Harpak, Z. Agur, Mutli-scale analysis of angiogenic dynamics and therapy, in: L. Preziosi (Ed.), Cancer Modelling and Simulation, Chapman \& Hall, CRC, London, 2003, pp. 185-219. 